\title{
Another Look on the Structure of Mountain Waves: A Spectral Perspective
}

\author{
Xin $\mathrm{Xu}^{\mathrm{a}}$, Shoujuan Shu ${ }^{\mathrm{a}}$, and Yuan Wang ${ }^{\mathrm{a}}$ \\ ${ }^{a}$ Key Laboratory of Mesoscale Severe Weather (Ministry of Education), School of \\ Atmospheric Sciences, Nanjing University, Nanjing 210023, P. R. China
}

(Submitted to Atmospheric Research)

March 5, 2017 


\begin{abstract}
Linear wave solutions in the spectral space are analyzed to help understand the structure of mountain waves. Nonrotating and hydrostatic waves generated in wind with directional shear past a circular bell-shaped mountain are studied. The power spectra of perturbed vertical velocity and pressure are symmetrically distributed about the orientation of dominant wave component, which bisects the angle between surface wind and local wind directions. The maximum power spectrum increases with the horizontal wind speed but decreases with the wind turning angle. The power spectra of potential temperature and horizontal velocity exhibit an asymmetric distribution except at the surface, which are infinite for the wave components normal to the mean wind. These large-amplitude perturbations of potential temperature and horizontal velocity are advected downstream and the waves finally break, giving rise to the occurrence of turbulent wakes at various heights. All the wavefields rotate with height in the same direction of the mean wind. However, the perturbed vertical velocity and pressure turn at a rate slower than that of horizontal velocity and potential temperature. The application of spectral analysis to the wave momentum flux is discussed, which helps explain the misalignment of wave momentum flux with the surface wind.
\end{abstract}

Keywords: gravity waves; wave structure; spectral analysis 


\section{Introduction}

Orographic gravity waves (OGWs) are excited as stably stratified flows interact with mountains on the earth. OGWs are capable of vertically transporting momentum from their source (i.e., orography) to the upper atmosphere, thus exerting an important influence on the mesospheric general circulation (Holton 1983). Mountain wave activities are also intimately related to many weather phenomena, some of which may cause casualties. For example, the breaking of OGWs is an importance source of clear air turbulence which threatens the safety of aviation. Wildfire may be induced in the leeside of high mountains through the well-known foehn effect (Smith 1985).

In the theoretical studies of OGWs, the wave equation is often solved in the spectral space, which is an ordinary differential equation. The spectral solution is then remapped to the physical space to examine the mountain wave structure. For example, Smith (1980) investigated the structure of nonrotating hydrostatic waves generated in constant wind over a three-dimensional (3D) circular bell-shaped mountain. While the remapping of spectral wave solution to the physical space offers many advantages, it has shortcomings as well. The remapped solution in the physical space often contains integrals that cannot be represented by elementary functions. As a result, they must be computed numerically (e.g., Teixeira and Miranda 2006; Xu et al. 2012, hereafter XWX12). It is not easy to understand the underlying physics according to complex integral expressions.

Since the physical solution can be viewed as a superposition of different Fourier harmonics, it is natural to study the spectral solution directly, i.e., spectral analysis. This spectral perspective has been adopted in the study of gravity wave momentum flux (WMF), which is closely related to the parameterization of gravity wave drag in 
numerical models (Kim et al. 2003). For instance, McFarlane (1987) parameterized the orographic WMF using a single wave along the surface wind direction. In contrast, Hines (1988) found that it was more appropriate to represent the WMF from isotropic terrain using two waves rather than a single one. By analyzing the WMF spectrum at the cloud top, Song and Chun (2005) developed a spectral parameterization scheme for non-orographic gravity wave drag.

In this work, the mountain wave structure generated in directionally sheared winds past a circular bell-shaped mountain is studied as an example from the spectral perspective. This kind of flow has already been investigated by Shutts (1998) through a combination of ray-tracing and stationary-phase methods. The wavefield was found to broaden with height, similar to the constant wind case (Smith 1980). Moreover, as the ambient wind turns with height, the wavefield also rotates in the same direction as the wind but at a relatively slower rate. The presence of directional wind shear causes a number of selective critical levels, at which the horizontal wave vector is normal to the local mean wind (Shutts 1995). Mountain waves are advected downstream on meeting the selective critical levels, which finally break and in consequence produce turbulent wakes at various altitudes (Broad 1999). The stationary-phase ray solution in Shutts (1998) has a simple form with no integral. Yet it is only valid far away from the wave source (i.e., mountain). In view of this, the Maslov's method was suggested in Broutman et al. (2002), which can avoid the ray-solution singularity directly above the terrain. However, the Maslov solution consists of an integral expression. As will be shown in this study, the OGW structural features can be readily understood from the viewpoint of spectral analysis.

The rest of this paper is organized as follows. Section 2 derives the spectral wave solutions for hydrostatic nonrotating mountain waves generated in directional wind 
varying linearly with height. The spectral solutions are analyzed in section 3 for a particular case of circular bell-shaped mountain. The application of spectral analysis to the WMF is presented in section 4. Finally, section 5 summarizes this paper with discussions.

\section{Linear wave solution}

According to the theory of two-dimensional (2D) Fourier transforms, the vertical velocity in the spectral space is

$$
\hat{w}(k, l, z)=(2)^{2} \int_{\infty}^{+\infty} \int_{\infty}^{+\infty} w(x, y, z) \exp [i(k x+l y)] d x d y
$$

where $\mathbf{K}=(k, l)$ is the horizontal wave vector, and $w(x, y, z)$ is the vertical velocity in the physical domain. Under the assumption of steady, adiabatic, inviscid, nonrotating, hydrostatic, and Boussinesq flow, the governing equation of spectral vertical velocity is given by (cf. Eq. (9) of XWX12),

$$
\widehat{w}_{z z}+m(z)^{2} \widehat{w}=0
$$

where $m^{2}=N^{2} K^{2} / \widehat{D}(z)^{2}$ is the squared vertical wave number, $K=|\mathbf{K}|, N$ is the Brunt-Väisälä frequency, and $\widehat{D}(z)=\mathbf{V}(z) \cdot \mathbf{K}$. (Hereafter, the subscript represents partial derivative unless otherwise stated or defined.) In deriving the above equation, the horizontally uniform mean wind is assumed to vary linearly with height, i.e.,

$$
\mathbf{V}(z)=\left(U_{0}+U_{z} z, V_{0}+V_{z} z\right)=|\mathbf{V}(z)|(\cos \psi(z), \sin \psi(z)),
$$

where $\mathbf{V}_{0}=\left(U_{0}, V_{0}\right)$ is the surface wind, $\mathbf{V}_{z}=\left(U_{z}, V_{z}\right)$ the vertical shear, and $\psi(z)$ is the azimuth of $\mathbf{V}(z)$. By virtue of the Frobenius method (e.g., Booker and Bretheron 1967), the analytical solution of the wave equation is (see XWX12)

$$
\widehat{W}(K, \varphi, z)=\widehat{W}(K, \varphi, 0) \sqrt{\frac{\widehat{D}(z)}{\widehat{D}_{0}}} \exp \left[i \operatorname{sgn}\left(\widehat{D}_{z}\right) \ln \frac{\widehat{D}(z)}{\widehat{D}_{0}} \sqrt{\frac{\mathrm{Ri}}{\cos ^{2}\left(\chi_{0}-\varphi\right)}-\frac{1}{4}}\right],
$$


where $\varphi$ is the azimuth of $\mathbf{K}$, namely, $\mathbf{K}=K(\cos \varphi, \sin \varphi), \widehat{D}_{0}=\mathbf{V}_{\mathbf{0}} \cdot \mathbf{K}, \widehat{D}_{z}=\mathbf{V}_{z} \cdot \mathbf{K}$, $\operatorname{sgn}(\cdot)$ is the sign function, $\mathrm{Ri}=N^{2} /\left|\mathbf{V}_{z}\right|^{2}$ is the mean flow Richardson number, and $\chi_{0}$ is the azimuth of wind vertical shear. At the lower boundary, it satisfies the free-slip condition $\widehat{w}(k, l, 0)=i \widehat{D}_{0} \hat{h}$, where $\hat{h}=\widehat{h}(K, \varphi)$ is the 2D Fourier transform of the mountain $h(x, y)$. Note that wave transmission above critical levels is excluded by assuming that gravity waves are totally absorbed at critical levels in the case of large Richardson numbers (Booker and Bretherton 1967).

Once the vertical velocity is obtained, one can readily derive other wave variables, such as horizontal velocity, pressure, and potential temperature

$$
\begin{array}{r}
\hat{u}=\frac{i}{K^{2}}\left(k \frac{\partial}{\partial z}-l \frac{k V_{z}-l U_{z}}{\widehat{D}}\right) \widehat{w}, \\
\hat{v}=\frac{i}{K^{2}}\left(l \frac{\partial}{\partial z}+k \frac{k V_{z}-l U_{z}}{\widehat{D}}\right) \widehat{w}, \\
\hat{p}=i \frac{\bar{\rho}}{K^{2}}\left(\widehat{D}_{z}-\widehat{D} \frac{\partial}{\partial z}\right) \widehat{w}, \\
\hat{\theta}=\frac{i}{\widehat{D}} \frac{\partial \bar{\theta}}{\partial z} \widehat{w},
\end{array}
$$

where $\bar{\rho}$ is the reference density, and $\bar{\theta}(z)$ is the base-sate potential temperature. These equations give the relative phases and amplitudes of different wave quantities, known as polarization relation (see Appendix).

\section{Results}

\section{a. Spectral analysis}

In accordance with Eqs. (5)-(8), the power spectra of perturbed vertical velocity, pressure, potential temperature, and horizontal velocity are given by,

$$
|\widehat{w}(K, \varphi, z)|^{2}=\left|\mathbf{V}_{\mathbf{0}}\right||\mathbf{V}(z)| \cos \left(\varphi-\psi_{0}\right) \cos [\varphi-\psi(z)] K^{2}|\hat{h}|^{2},
$$




$$
\begin{aligned}
& |\hat{p}(K, \varphi, z)|^{2}=\bar{\rho}^{2} \frac{N^{2}}{K^{2}}|\widehat{w}(K, \varphi, z)|^{2} \\
& |\hat{\theta}(K, \varphi, z)|^{2}=\left(\frac{\partial \bar{\theta}}{\partial z}\right)^{2} \frac{\widehat{D}_{0}}{\widehat{D}(z)}|\hat{h}|^{2} \\
& |\widehat{u}(K, \varphi, z)|^{2}=\left|\mathbf{V}_{z}\right|^{2} \frac{\widehat{D}_{0}}{\widehat{D}(z)}\left[\operatorname{Ricos}{ }^{2} \varphi+\cos \chi_{0} \sin \varphi \sin \left(\varphi-\chi_{0}\right)\right]|\hat{h}|^{2}, \\
& |\hat{v}(K, \varphi, z)|^{2}=\left|\mathbf{V}_{z}\right|^{2} \frac{\widehat{D}_{0}}{\widehat{D}(z)}\left[\operatorname{Risin}^{2} \varphi-\sin \chi_{0} \cos \varphi \sin \left(\varphi-\chi_{0}\right)\right]|\hat{h}|^{2},
\end{aligned}
$$

To facilitate the spectral analysis, we use a circular bell-shaped orography, i.e.,

$$
h(x, y)=h_{m}\left[1+\left(x / r_{a}\right)^{2}+\left(y / r_{a}\right)^{2}\right]^{-3 / 2},
$$

where $h_{m}$ and $r_{a}$ are the mountain height and half width, respectively. (It should be noted that the results obtained below are valid for any axisymmetric mountain.) The spectrum of the isotropic bell-shaped mountain only depends on the horizontal wave vector magnitude

$$
\hat{h}(K)=(2 \pi)^{-1} h_{m} a^{2} e^{-r_{a} K} .
$$

In this case, the partial derivative of $|\widehat{w}|^{2}$ with respect to $\varphi$ (namely, the orientation of horizontal wave vector) is

$$
\frac{\partial}{\partial \varphi}\left(|\widehat{w}(K, \varphi, z)|^{2}\right)=-\left|\mathbf{V}_{\mathbf{0}}\right||\mathbf{V}(z)| K^{2}|\widehat{h}|^{2} \sin ^{2}\left[2 \varphi-\psi_{0}-\psi(z)\right] .
$$

Evidently, Eq. (16) equals to zero at

$$
\varphi_{\max }(z)=\left[\psi_{0}+\psi(z)\right] / 2
$$

which bisects the angle between surface wind and local wind directions (i.e., the wind at height $z$ ). For given $K$ and $z,|\widehat{w}|^{2}$ has a peak value at $\varphi_{\max }(z)$ owing to its negative second derivative there (not shown). Substitution of Eq. (17) into Eq. (9) yields the maximum power spectrum of vertical velocity

$$
|\widehat{w}(K, z)|_{\max }^{2}=\left|\mathbf{V}_{\mathbf{0}}\right||\mathbf{V}(z)| \cos ^{2}\left[\frac{\psi(z)-\psi_{0}}{2}\right] K^{2}|\hat{h}|^{2}
$$


It increases with the horizontal wind speed, because stronger waves are generated by greater horizontal wind. Moreover, the maximum power spectrum of vertical velocity decreases with the wind turning angle $\left|\psi(z)-\psi_{0}\right|$. (Note that the wind turning angle is between $(0, \pi)$ such that $\left|\psi(z)-\psi_{0}\right| / 2$ is in the range $(0, \pi / 2)$.) This is owing to that the power spectrum of vertical velocity is focused near $\psi_{0}$ at the surface. Therefore, the smaller angle the wind turns with height, the more the $\varphi_{\max }$ is close to $\psi_{0}$. It is also easy to prove that the vertical velocity power spectrum is symmetric with respect to $\varphi_{\max }(z)$. Thus the wavefield of vertical velocity is dominated by the wave component in the direction of $\varphi_{\max }(z)$. Since $\varphi_{\max }(z)$ changes accordingly with $\psi(z)$, the wavefield is expected to turn with height in the same direction as the mean wind, although it only amounts for half the rotation of the wind. As seen from Eq. (10), the power spectrum of pressure perturbation has the same distribution about the orientation of horizontal wave vector.

The power spectrum of perturbed potential temperature is the same as that of terrain except for a factor of $\left(\frac{\partial \bar{\theta}}{\partial z}\right)^{2}$ at the surface (see Eq. (11)). In contrast, it is dominated by $\widehat{D}_{0} / \widehat{D}(z)$ at high altitudes. The power spectrum becomes infinite at $\widehat{D}(z)=0$ where the governing wave equation is singular. This occurs either as $\mathbf{V}(z)$ vanishes (i.e., the classic critical level, Booker and Bretherton 1967) or the horizontal wave vector is perpendicular to the mean wind (i.e, selective critical level, Shutts 1995). In the directional wind case studied, the mean wind cannot become zero such that the power spectrum is focused near the wave component perpendicular to the mean wind, i.e., $\varphi_{c}(z)=\psi(z)-\pi / 2$ and $\varphi_{c}(z)=\psi(z)+\pi / 2$. Because $\varphi_{c}(z)$ varies with $\psi(z)$, the wavefield of potential temperature is expected to rotate with height following the wind, at a rate faster than that of vertical velocity. The power spectra of perturbed horizontal velocity $u$ and $v$ are somewhat more complicated, but they are dominated 
by $\widehat{D}_{0} / \widehat{D}(z)$ as well. In the situation of nonrotating hydrostatic OGWs, the group velocity $\mathbf{c}_{\mathrm{g}}=\left(c_{\mathrm{g} x}, c_{\mathrm{g} y}, c_{\mathrm{g} z}\right)=\left(\frac{\partial \omega}{\partial k}, \frac{\partial \omega}{\partial l}, \frac{\partial \omega}{\partial m}\right)$ can be expressed as

$$
\left(c_{\mathrm{g} x}, c_{\mathrm{g} y}\right)=|\mathbf{V}| \sin [\Psi(z)-\varphi](-\sin \varphi, \cos \varphi), \quad c_{\mathrm{g} z}=N K / m^{2},
$$

where $\omega=U k+V l-N K / m$ is the wave frequency. For waves encountering selective critical level, i.e., $\varphi=\varphi_{c}(z)$, the horizontal group velocity is parallel to the mean wind, with zero vertical group velocity (Shutts 1998). In other words, these waves are advected downstream at selective critical levels. Given the infinitely large power spectra of perturbed potential temperature and horizontal velocity at $\varphi_{c}(z)$, it suggests downstream trailing of large-amplitude disturbances in their wavefields (Shutts and Gadian 1999).

\section{b. An example}

The height and half width of the circular bell-shaped mountain are set to $h_{\mathrm{m}}=10$ $\mathrm{m}$ and $r_{\mathrm{a}}=20 \mathrm{~km}$, respectively. A directionally sheared wind backing with height is adopted with $U_{0}=8 \mathrm{~m} \mathrm{~s}^{-1}, V_{0}=0, U_{z}=0, V_{\mathrm{z}}=5 \times 10^{-3} \mathrm{~s}^{-1}$, i.e., $\psi_{0}=0, \chi_{0}=\pi / 2$. The atmosphere is of a constant buoyancy frequency $N=0.01 \mathrm{~s}^{-1}$. The base-sate potential temperature is $288 \mathrm{~K}$ at the surface. Under these conditions, the Froude number is $\mathrm{Fr}$ $=\left|\mathbf{V}_{0}\right| / N h_{0}=80>>1$, indicating linear waves (e.g., Miranda and James 1992). The hydrostatic assumption is also satisfied given that $N a /\left|\mathbf{V}_{0}\right|=25>1$ (Smith 1979).

Figure 1a shows the distribution of power spectrum with respect to the horizontal wave vector orientation for vertical velocity at $z=2.5 \mathrm{~km}$ and $z=5 \mathrm{~km}$ respectively. Note that the power spectrum is scaled with respect to $K^{2}|\hat{h}|^{2}$. Thus it is independent of the wave vector magnitude $K$. The wave solution in the physical space is recovered via inverse 2D Fourier transform, for example, 


$$
w(R, \quad, z)=2_{{ }_{L}}^{U} \hat{w}(K, \quad, z) e^{i K R \cos (\quad)} K d K d
$$

where the polar coordinates $(x, y)=R(\cos \Psi$, $\sin \Psi)$ with $R=\left(x^{2}+y^{2}\right)^{1 / 2}$ are adopted. For directionally sheared winds backing with height, the lower and upper limits of the integral are $\varphi_{L}=\psi(z)-\pi / 2$ and $\varphi_{U}=\psi_{0}+\pi / 2$, with $\psi_{0}$ being the azimuth of surface wind (XWX12). As for veering winds, $\varphi_{L}=\psi_{0}-\pi / 2$ and $\varphi_{U}=\psi(z)+\pi / 2$. Hereafter, $\varphi_{L}$ and $\varphi_{U}$ are termed as the left and right tails of the spectrum. The power spectrum is symmetric about $\varphi_{\max }(z)$ where the maximum response occurs. As shown in Figs. 2a and $2 b$, the primary wave front is generally normal to the direction of $\varphi_{\max }(z)$. The largest power spectrum at $z=5 \mathrm{~km}$ is greater than that at $z=2.5 \mathrm{~km}$, because the wind speed increases with height for the ambient wind used. Moreover, the power spectrum narrows with height. It occurs as the waves at the spectrum left tail are continuously filtered on meeting the selective critical levels during upward propagation. (For winds veering with height, it will be the waves at the right tail that undergo selective critical level absorption.) Because of the continuous critical level filtering, the waves become increasingly monochromatic. The narrowing of the wave spectrum and the amplitude amplification subsequently cause a concentration of the power spectrum with height. In other words, the wave component in the direction $\varphi_{\max }(z)$ becomes more and more dominant, which gives rise to the widening of the wavefield with height. Additionally, as expected from spectral analysis, the wavefield turns anticlockwise with height, in the same direction as the background wind.

Figure $1 \mathrm{~b}$ displays the power spectrum of potential temperature scaled about $|\hat{h}|^{2}$. It also narrows with height, but exhibits an asymmetric distribution with respect to the dominant horizontal wave vector orientation. The power spectrum becomes infinite at the left tail where $\varphi(z)=\varphi_{c}(z)=\psi(z)-\pi / 2$, but drops rapidly toward the right tail. As 
a consequence, the dominant wave front of potential temperature is nearly parallel to the local wind, turning anticlockwise with height (Figs. $2 \mathrm{c}$ and $2 \mathrm{~d}$ ). These waves can propagate downstream for a long distance, in particular at $z=5 \mathrm{~km}$ (Fig. 2d). (Note that the wavefield of potential temperature is displayed in an area much larger than that of vertical velocity because it is more spread spatially.) It is in large contrast to vertical velocity, the power spectrum of which vanishes at $\varphi_{c}(z)$ (see Fig. 1a; Nappo 2012), leading to weak perturbations in the mountain wake (Figs. 2a and 2b).

Figure 3 presents the power spectra of horizontal velocity scaled about $|\hat{h}|^{2}$ at $z$ $=5 \mathrm{~km}$, with their wavefields shown in Fig. 4 . Similar to that of potential temperature, the power spectra of horizontal velocity are also focused near the left tail and decrease rapidly rightward (Fig. 3). The most significant wave fronts are generally in parallel with the local mean flow, trailing downstream well into the far-field wake (Fig. 4). In theory, large perturbation of horizontal velocity will result in shear instability, while convective instability can be induced by large potential temperature perturbation. In this regard, mountain waves are expected to become unstable and break, leading to the production of turbulence (Broad 1999; Guarino et al. 2016). This helps explain the turbulent nature of the asymptotic wake found in previous studies (e.g., Doyle and Jiang 2006; Lane 2009).

\section{Application to wave momentum flux}

While spectral analysis can help understand the structural features of OGWs, it can also be used in the parameterization of gravity wave drag in large-scale numerical models (Kim et al. 2003). Taking the orographic gravity wave drag (OGWD) as an example, current parameterization schemes do not take into account the effect of 3D selective critical level absorption. Moreover, the oblique propagation of 3D mountain 
waves (i.e., the waves propagate both vertically and horizontally) is not considered either (Kalisch et al. 2014). In order to represent these 3D effects, spectral schemes addressing the wave components individually are required (Hasha et al. 2008; Song and Chun 2008). The accuracy of a spectral parameterization scheme is at the cost of computational time, because it will become time consuming when a large number of wave components are taken into account. To facilitate the implementation of spectral parameterization schemes, it is helpful to pre-analyze the WMF spectrum to figure out the most remarkable wave components.

Again, the OGW generated in directional shear wind past a circular bell-shaped mountain is investigated as an example. From XWX12, the surface WMF in this case can be expressed as follows (cf. their Eq. (25))

$$
\mathbf{F}_{0}=2 \mathbf{F}_{c s t}{ }_{0}^{0^{+} / 2}(\cos , \sin ) \cos (\quad 0) \sqrt{1 \frac{\cos ^{2}\left(\begin{array}{l}
0 \\
4 \mathrm{Ri}
\end{array}\right)}{d}} d
$$

where $\mathbf{F}_{c s t}=0.25 \bar{\rho} N r_{a} h_{m}^{2} \mathbf{V}_{\mathbf{0}}$ is the WMF generated in constant wind. Note that this equation is only valid at surface, because the WMF in directional wind changes with height due to selective critical level absorption. From Eq. (21), the power spectrum of surface WMF is given by

$$
\left|\widehat{\mathbf{F}_{0}}(\varphi)\right|^{2}=\cos ^{2}\left(\varphi-\psi_{0}\right)\left[1-\frac{\cos ^{2}\left(\chi_{0}-\varphi\right)}{4 \mathrm{Ri}}\right]
$$

which has been scaled with respect to $2\left|\mathbf{F}_{c s t}\right|$ for simplicity. Defining a new variable $\delta=\varphi-\psi_{0}$, which is in the range of $\delta \in(-\pi / 2, \pi / 2)$, the above equation can be rewritten as

$$
\left|\widehat{\mathbf{F}_{0}}(\delta, \Theta, \mathrm{Ri})\right|^{2}=\cos ^{2} \delta\left[1-\frac{\cos ^{2}(\Theta-\delta)}{4 \mathrm{Ri}}\right]
$$


where $\Theta=\chi_{0}-\psi_{0}$ is the maximum turning angle of the mean wind with height, lying in the range of $\Theta \in[0, \pi]$. Note that in the situation of $\Theta=0$ or $\Theta=\pi$, the directionally sheared wind is reduced to a unidirectional wind.

Figure 5 shows the distribution of $\left|\widehat{\mathbf{F}_{0}}(\delta, \Theta, \mathrm{Ri})\right|^{2}$ in the $\delta-\Theta$ space for different Richardson numbers. The WMF power spectrum is focused near $(\delta, \Theta)=$ $(0, \pi / 2)$, especially at small Ri. At moderate-to-large Ri, the WMF is concentrated between $\delta=(-\pi / 3, \pi / 3)$ for all $\Theta$. However, at given $\Theta$ (except $\Theta=\pi / 2$ ) the WMF power spectrum is asymmetric with respect to $\delta=0$, i.e., the horizontal wave vector in the direction of surface wind. This results in a misalignment of surface WMF with the wind, even in the case of isotropic mountain, which has been noticed in Teixeira et al. (2004, hereafter TMV04). On substituting the linearly sheared wind Eq. (3) into the second-order Wentzel-Kramers-Brillouin (WKB) solution of TMV04 (cf. their Eqs. (50 and (51)), the surface WMF is given by

$$
\begin{aligned}
& F_{x}=F_{c s t x}\left[1-\frac{1}{32 R i}\left(3-2 \frac{\sin \chi_{0}}{\cos \psi_{0}} \sin \Theta\right)\right], \\
& F_{y}=F_{\text {csty }}\left[1-\frac{1}{32 R i}\left(3+2 \frac{\cos \chi_{0}}{\sin \psi_{0}} \sin \Theta\right)\right],
\end{aligned}
$$

where $\mathbf{F}_{c s t}$ is the constant-wind WMF. Clearly, the wind vertical shear gives different corrections to the $x$ and $y$ components of the WMF, except $\Theta=\pi / 2$. The asymmetry increases as the Richardson number decreases (Fig. 5). It is due to that, for the wind profile Eq. (3) used, the surface WMF varies proportionally to $\mathrm{Ri}^{-1}$ (see Eqs. (24) and (25)).

Herein we only consider a simple case of circular bell-shaped mountain. Spectral analysis can also be applied to anisotropic terrain, such as elliptical mountain (Phillps 1984; Teixeira and Miranda 2006) and realistic orography. For example, Eckermann et al. (2006), Pulido and Rodas (2011), and Jiang et al. (2014) have tried to mimic the 
mountain waves in reality using different approaches, all of which are on the basis of superposition of a number of wave components.

\section{Summary and discussion}

From the viewpoint of spectral analysis, this study provides another look on the structure of orographic gravity waves (OGWs) generated in wind with directional shear over an isolated circular bell-shaped mountain. Under the assumption of steady, adiabatic, nonrotating, hydrostatic, and Boussinesq flow, the linearized wave equation governing the vertical velocity is analytically solved in the spectral space. Other wave variables, such as pressure, potential temperature and horizontal velocity, are obtained according to the polarization relation of plane wave.

The power spectra of vertical velocity and pressure are symmetrically distributed about the dominant horizontal wave vector in the direction of $\varphi_{\max }(z)=\left[\psi_{0}+\psi(z)\right] / 2$, with $\psi_{0}$ and $\psi(z)$ denoting the directions of mean wind at surface and height $z$. The maximum power spectrum, which occurs at $\varphi_{\max }(z)$, increases with the horizontal wind speed but decreases with the wind turning angle. By contrast, the power spectra of perturbed potential temperature and horizontal velocities exhibit an asymmetric distribution except at the surface. Both are infinite at $\varphi_{c}(z)$, which is perpendicular to the local mean wind, i.e., the wave components encountering selective critical levels.

Because the orientation of dominant wave changes accordingly with the mean wind direction, the wavefield turns with height in the same direction as the mean wind. However, the wavefields of vertical velocity and pressure rotate at a rate slower than that of horizontal velocity and potential temperature. Moreover, OGWs are advected downstream at selective critical levels. Thus large-amplitude perturbations are found in the far field for potential temperature and horizontal velocity. The development of 
convective (shear) instability induced by the large potential temperature (horizontal velocity) perturbations can result in the occurrence of wave breaking, which in turn gives rise to a turbulent mountain wake.

The application of spectral analysis to the surface wave momentum flux (WMF) is also discussed. The power spectrum of WMF is focused near $(\delta, \Theta)=(0, \pi / 2)$ at small Richardson numbers, where $\delta$ is the angle between horizontal wave vector and surface wind, and $\Theta$ is the maximum turning angle of the mean wind with height. At moderate-to-large Richardson numbers, the power spectrum of WMF is concentrated between $-\pi / 3<\delta<\pi / 3$. At given $\Theta$ that is not equal to $\pi / 2$, the WMF spectrum is asymmetrically distributed about $\delta=0$. This helps explain why the WMF is not aligned with the surface wind in the case of directionally sheared wind.

The present study mainly focuses on the mountain waves generated in directional wind over an idealized terrain. The application of spectral analysis to more general mountain-wave cases will be addressed in our future research. In addition to the understanding of mountain-wave structure, spectral analysis can also be used to study the influence of mountain waves on other weather systems. For example, Torre et al. (2011, 2015) studied the relation between the occurrence of hailstorms and mountain waves near the Southern Andes. The continuous wavelet transform was adopted to detect the main oscillation modes of mountain waves. Their results showed that the updrafts of dominant waves are able to provide the necessary kinetic energy to overcome the convection inhibition and thus trigger convective storms. This issue will also be studied in the future. 
Acknowledgements. The authors thank the anonymous reviewers for their constructive comments and suggestions. This work is mainly supported by National Fundamental Research 973 Program of China (2015CB452801), National Science Foundation of China (41505046), and Fundamental Research Funds for the Central Universities at Nanjing University (020714380008). 


\section{Appendix A: Derivation of polarization relation}

The linearized governing equations for adiabatic, non-rotating, Boussinesq and hydrostatic atmosphere are given by

$$
\begin{aligned}
\bar{\rho} D u+\bar{\rho} U_{z} w+\frac{\partial p}{\partial x} & =0, \\
\bar{\rho} D v+\bar{\rho} V_{z} w+\frac{\partial p}{\partial y} & =0, \\
\rho g+\frac{\partial p}{\partial z} & =0, \\
\Delta+\frac{\partial w}{\partial z} & =0, \\
D \theta+w \frac{\partial \bar{\theta}}{\partial z} & =0,
\end{aligned}
$$

where $D=U \frac{\partial}{\partial x}+V \frac{\partial}{\partial y}$, and $\Delta=\frac{\partial u}{\partial x}+\frac{\partial v}{\partial y}$ is the horizontal divergence. On applying the 2D Fourier transform onto Eq. (A5), it is easy to obtain the polarization relation between potential temperature and vertical velocity, i.e.,

$$
\hat{\theta}=\frac{i}{\widehat{D}} \frac{\partial \bar{\theta}}{\partial z} \widehat{w}
$$

As for pressure, first taking $\partial(\mathrm{A} 1) / \partial x$ and $\partial(\mathrm{A} 2) / \partial y$, then adding the resultant two equations yields

$$
\hat{p}=i \frac{\bar{\rho}}{K^{2}}\left(\widehat{D}_{z}-\widehat{D} \frac{\partial}{\partial z}\right) \widehat{W} .
$$

To obtain the relation between horizontal and vertical velocities, we first derive the vertical vorticity equation from the horizontal momentum equations,

$$
D \xi+\left(V_{z} \frac{\partial w}{\partial x}-U_{z} \frac{\partial w}{\partial y}\right)=0,
$$

where $\xi=\frac{\partial v}{\partial x}-\frac{\partial u}{\partial y}$ is the vertical vorticity. According to the definition of horizontal divergence and vertical vorticity, we have

$$
\nabla^{2} u=\frac{\partial \Delta}{\partial x}-\frac{\partial \xi}{\partial y},
$$




$$
\nabla^{2} v=\frac{\partial \xi}{\partial x}+\frac{\partial \Delta}{\partial y}
$$

Applying 2D Fourier transform onto the above two equations leads to

$$
\begin{gathered}
\hat{u}=\frac{i}{K^{2}}\left(k \frac{\partial}{\partial z}-l \frac{k V_{z}-l U_{z}}{\widehat{D}}\right) \widehat{w}, \\
\hat{v}=\frac{i}{K^{2}}\left(l \frac{\partial}{\partial z}+k \frac{k V_{z}-l U_{z}}{\widehat{D}}\right) \widehat{w} .
\end{gathered}
$$




\section{References}

Booker, J. R., and Bretherton, F. P., 1967. The critical layer for internal gravity waves in a shear flow. J. Fluid Mech., 27, 513-539.

Broad, A. S., 1995. Linear theory of momentum fluxes in 3-D flows with turning of the mean wind with height. Q. J. R. Meteorol. Soc., 121, 1891-1902.

Broad, A. S., 1999. Do orographic gravity wave break in flows with uniform wind direction turning with height. Q. J. R. Meteorol. Soc., 125, 1696-1714.

Broutman, D., Rottman, J. W., and Eckermann, S. D., 2002. Maslov's method for stationary hydrostatic mountain waves. Q. J. R. Meteorol. Soc., 128, 1159-1171.

Eckermann, S. D., Broutman, D., Ma, J., and Lindeman, J., 2006. Fourier-ray modeling of short-wavelength trapped lee waves observed in infrared satellite imagery near Jan Mayen. Mon. Wea. Rev., 134, 2830-2848.

Doyle, J. D., and Jiang Q., 2006. Observations and numerical simulations of mountain waves in the presence of directional wind shear. Quart. J. Roy. Meteor. Soc., 132, 1877-1905.

Guarino, M. V., Teixeira, M. A. C. and Ambaum, M. H. P., 2016. Turbulence generation by mountain wave breaking in flows with directional wind shear. Quart. J. Roy. Meteor. Soc., 142, 2715-2726.

Hasha, A., Bühler, O., and Scinocca, J., 2008. Gravity wave refraction by threedimensionally varying winds and the global transport of angular momentum. J. Atmos. Sci., 65, 2892-2906.

Holton, J. R., 1983. The influence of gravity wave breaking on the general circulation of the middle atmosphere. J. Atmos. Sci., 40, 2497-2507. 
Jiang, Q., Reinecke, A., and Doyle, J. D., 2014. Orographic wave drag over the Southern Ocean: A linear theory perspective. J. Atmos. Sci., 71, 4235-4252.

Kalisch, S., Preusse, P., Ern, M., Eckermann, S. D., and Riese, M., 2014. Differences in gravity wave drag between realistic oblique and assumed vertical propagation. J. Geophys. Res. Atmos., 119, 10081-10099.

Kim, Y. J., Eckermann, S. D., and Chun, H. Y., 2003. An overview of the past, present and future of gravity-wave drag parametrization for numerical climate and weather prediction models. Atmos.-Ocean, 41, 65-98.

Lane, T. P., Doyle J. D., Sharman R. D., Shapiro M. A., and Watson C. D., 2009. Statistics and dynamics of aircraft encounters of turbulence over Greenland. Mon. Wea. Rev., 137, 2687-2702.

Lott, F., and Miller, M., 1997. A new sub-grid orographic drag parameterization: Its formulation and testing. Quart. J. Roy. Meteor. Soc., 123, 101-127.

Miranda, P. M. A., and James, I. N., 1992. Non-linear three-dimensional effects on gravity-wave drag: splitting flow and breaking waves. Q. J. R. Meteorol. Soc., $118,1057-1081$

Nappo, C., 2012. An introduction to atmospheric gravity waves. second edition. Academic press, 400pp.

Phillips, D. S., 1984. Analytical surface pressure and drag for linear hydrostatic flow over three-dimensional elliptical mountains. J. Atmos. Sci., 41, 1073-1084.

Pulido, M., and Rodas, C., 2011. A higher-order ray approximation applied to orographic waves: Gaussian beam approximation. J. Atmos. Sci., 68, 46-60.

Shutts, G. J., 1995. Gravity-wave drag parametrization over complex terrain: The effect of critical level absorption in directional wind-shear. Q. J. R. Meteorol. Soc., 121, 1005-1021. 
Shutts, G. J., 1998. Stationary gravity-wave structure in flows with directional wind shear. Q. J. R. Meteorol. Soc., 124, 1421-1442.

Shutts, G. J., and Gadian A., 1999. Numerical simulations of orographic gravity waves in flows which back with height. Quart. J. Roy. Meteor. Soc., 125, 27432765.

Smith, R. B., 1980. Linear theory of stratified flow past an isolated mountain. Tellus, $32,348-364$.

Smith, R. B., 1985. On severe downslope winds. J. Atmos. Sci., 42, 2597-2603.

Song, I.-S., and Chun, H.-Y., 2005. Momentum flux spectrum of convectively forced internal gravity waves and its application to gravity wave drag parameterization. Part I: Theory. J. Atmos. Sci., 62, 107-124.

Song, I.-S., and Chun, H.-Y., 2008. A Lagrangian spectral parameterization of gravity wave drag induced by cumulus convection. J. Atmos. Sci., 65, 1204-1224.

Teixeira, M. A. C., Miranda, P. M. A., 2006. A linear model of gravity wave drag for hydrostatic sheared flow over elliptical mountains. Q. J. R. Meteorol. Soc., 132, $2439-2458$.

Teixeira, M. A. C., Miranda, P. M. A., and Valente, M. A., 2004. An analytical model of mountain wave drag for wind profiles with shear and curvature. J. Atmos. Sci., $61,1040-1054$.

Torre, A. de la, Hierro, R., Llamedo, P., Rolla, A., and Alexander, P., 2011. Severe hailstorms near Southern Andes in the presence of mountain waves. Atmos. Res., $101,112-123$.

Torre, A. de la, Pessano, H., Hierro, R., Santos, J. R., Llamedo, P., and Alexander, P., 2015. The influence of topography on vertical velocity of air in relation to severe storms near the Southern Andes Mountains. Atmos. Res., 156, 91-101. 
Xu, X., Wang, Y., and Xue, M., 2012. Momentum flux and flux divergence of gravity waves in directional shear flows over three-dimensional mountains. J. Atmos. Sci., 69, 3733-3744. 


\section{List of figure captions}

Fig. 1. Distribution of scaled power spectrum with respect to the horizontal wave vector orientation (unit: $\pi$ ) at the height of $z=2.5 \mathrm{~km}$ (solid blue) and $z=5 \mathrm{~km}$ (solid red) for (a) vertical velocity and (b) potential temperature. The dashed lines in (a) and (b) indicate the position of $\varphi_{\max }(z)$ and $\varphi_{\mathrm{c}}(z)$ respectively.

Fig. 2. Perturbation fields of (top) vertical velocity (unit: $\mathrm{m} \mathrm{s}^{-1}$ ) and (bottom) potential temperature (unit: K) at the height of $z=2.5 \mathrm{~km}$ (left) and $z=5 \mathrm{~km}$ (right). The black circle represents the circular bell-shaped topography. The black lines denote the mean flow direction, while the purple lines in (a) and (b) indicate the direction of $\varphi_{\max }(z)$. The $x$ and $y$ coordinates are normalized with respect to the mountain half width.

Fig. 3. Distribution of scaled power spectrum with respect to the horizontal wave vector orientation (unit: $\pi$ ) at the height of $z=5 \mathrm{~km}$ for horizontal velocity $u$ (blue) and $v\left(\right.$ red) respectively. The dashed line denotes the position of $\varphi_{\mathrm{c}}(z)$.

Fig. 4. Perturbation fields of horizontal velocity (a) $u$ (unit: $\mathrm{m} \mathrm{s}^{-1}$ ) and (b) $v$ (unit: $\mathrm{m}$ $\mathrm{s}^{-1}$ ) at the height of $z=5 \mathrm{~km}$. The black circle represents the circular bell-shaped topography. The black lines denote the mean flow direction. The $x$ and $y$ coordinates are normalized with respect to the mountain half width.

Fig. 5. Distribution of scaled power spectrum of wave momentum flux at (a) $\mathrm{Ri}=$ 0.25, (b) $\mathrm{Ri}=0.5$, (c) $\mathrm{Ri}=1$, and (d) $\mathrm{Ri}=4$. 

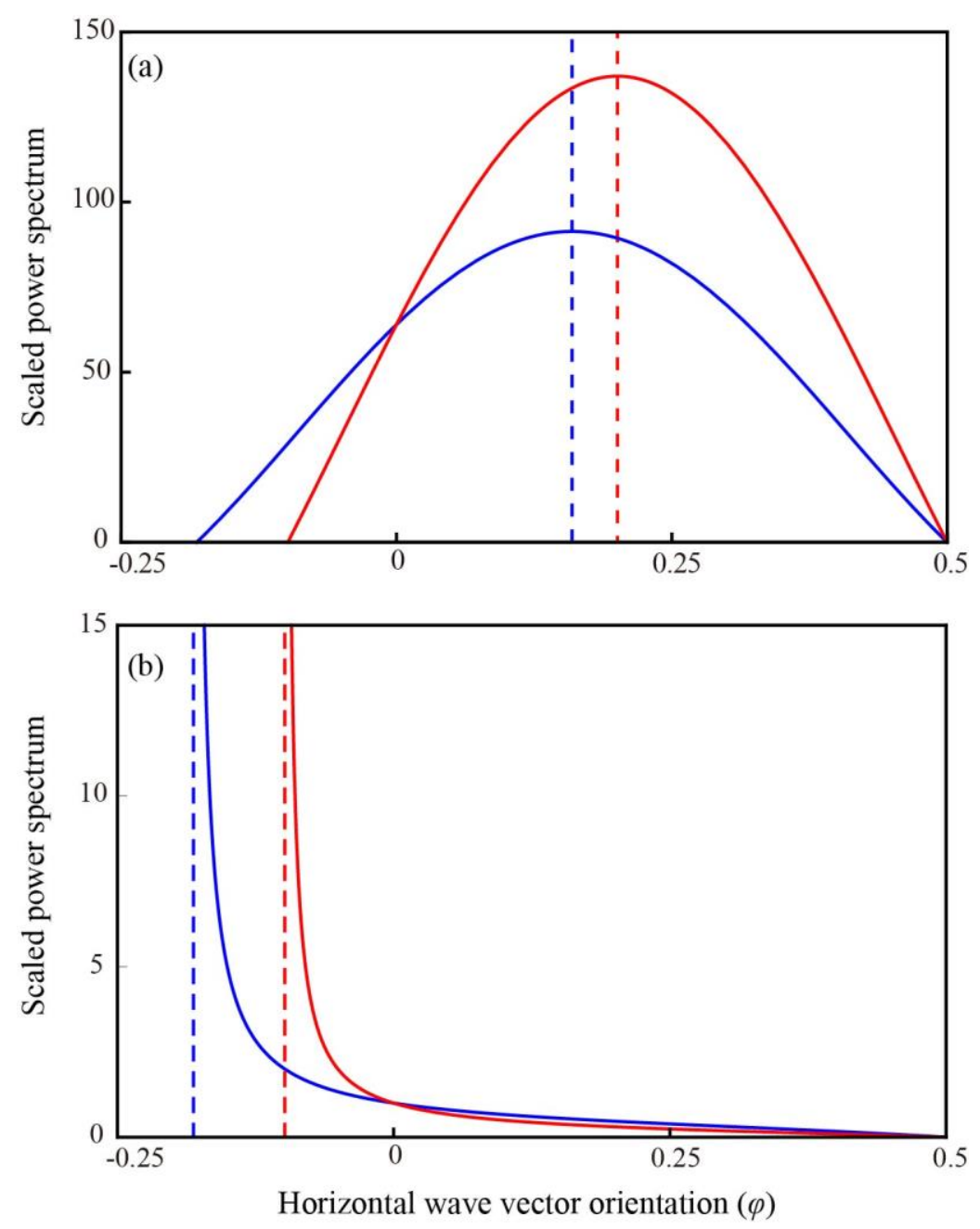

Fig. 1. Distribution of scaled power spectrum with respect to the horizontal wave vector orientation (unit: $\pi$ ) at the height of $z=2.5 \mathrm{~km}$ (solid blue) and $z=5 \mathrm{~km}$ (solid red) for (a) vertical velocity and (b) potential temperature. The dashed lines in (a) and (b) indicate the position of $\varphi_{\max }(z)$ and $\varphi_{c}(z)$ respectively. 

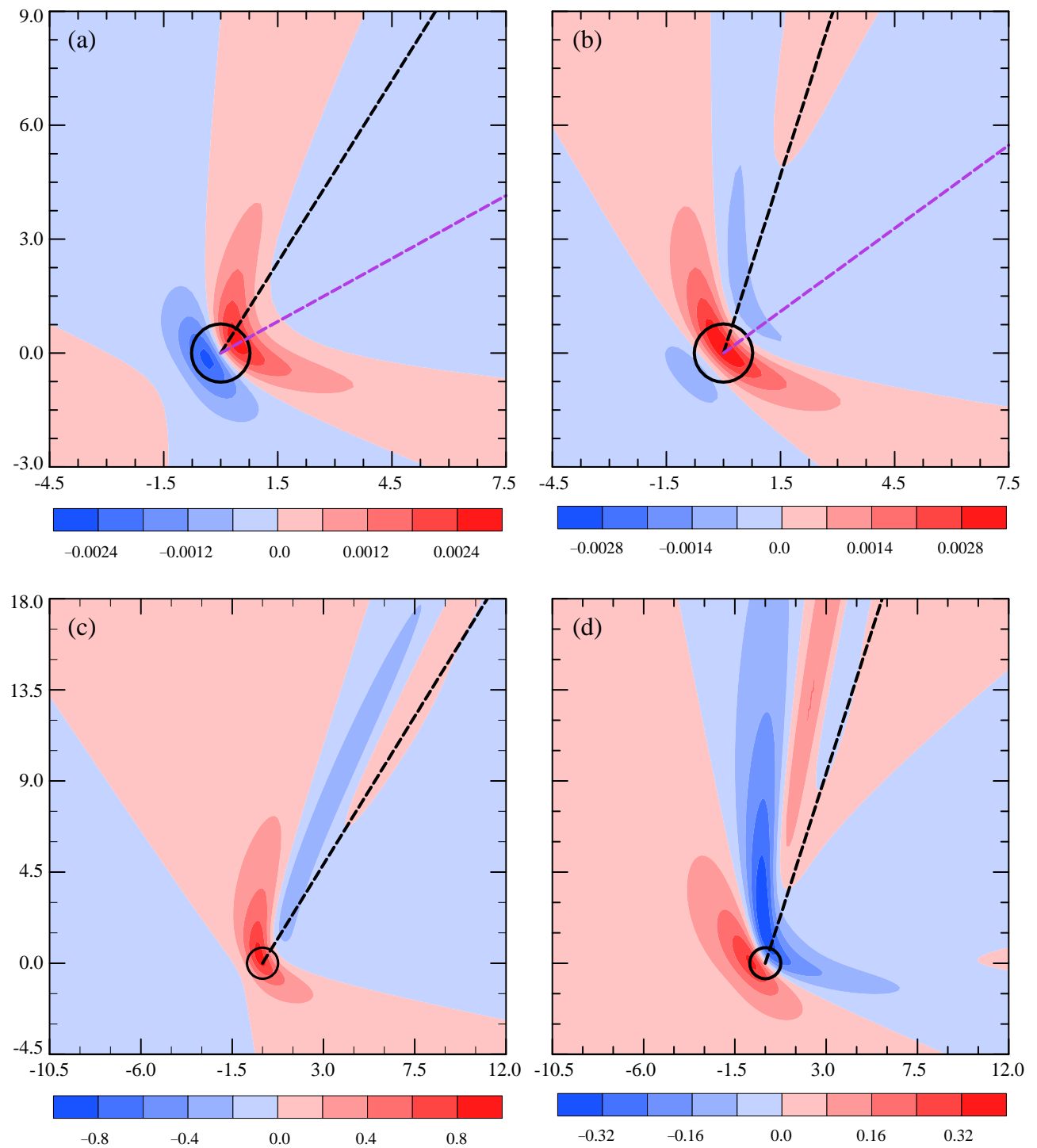

Fig. 2. Perturbation fields of (top) vertical velocity (unit: $\mathrm{m} \mathrm{s}^{-1}$ ) and (bottom) potential temperature (unit: K) at the height of $z=2.5 \mathrm{~km}$ (left) and $z=5 \mathrm{~km}$ (right). The black circle represents the circular bell-shaped topography. The black lines denote the mean flow direction, while the purple lines in (a) and (b) indicate the direction of $\varphi_{\max }(z)$. The $x$ and $y$ coordinates are normalized with respect to the mountain half width. 


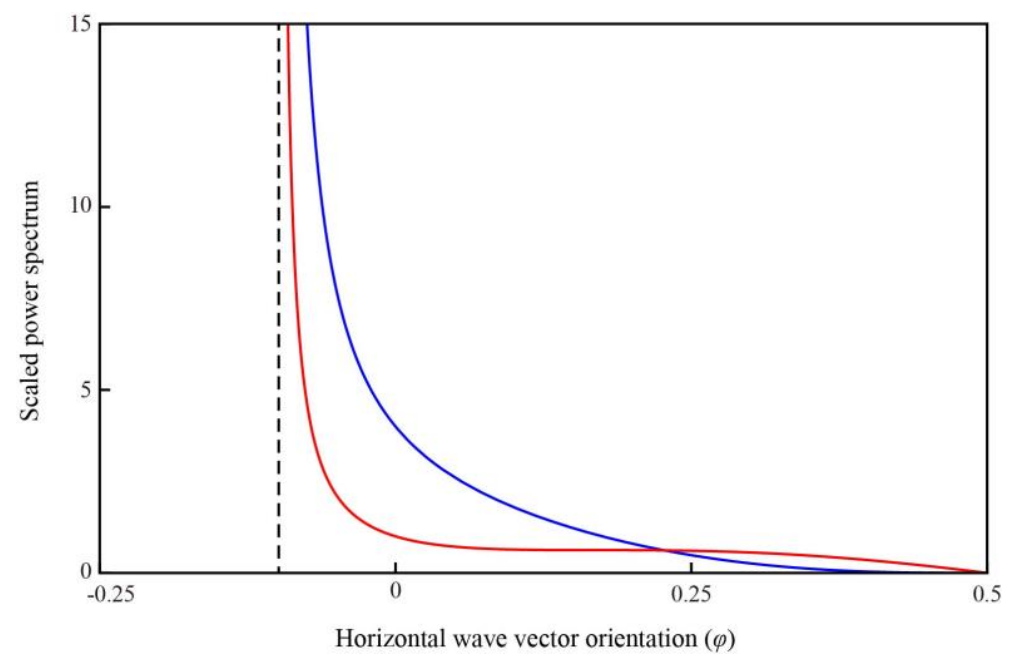

Fig. 3. Distribution of scaled power spectrum with respect to the horizontal wave vector orientation (unit: $\pi$ ) at the height of $z=5 \mathrm{~km}$ for horizontal velocity $u$ (blue) and $v$ (red) respectively. The dashed line denotes the position of $\varphi_{\mathrm{c}}(z)$. 

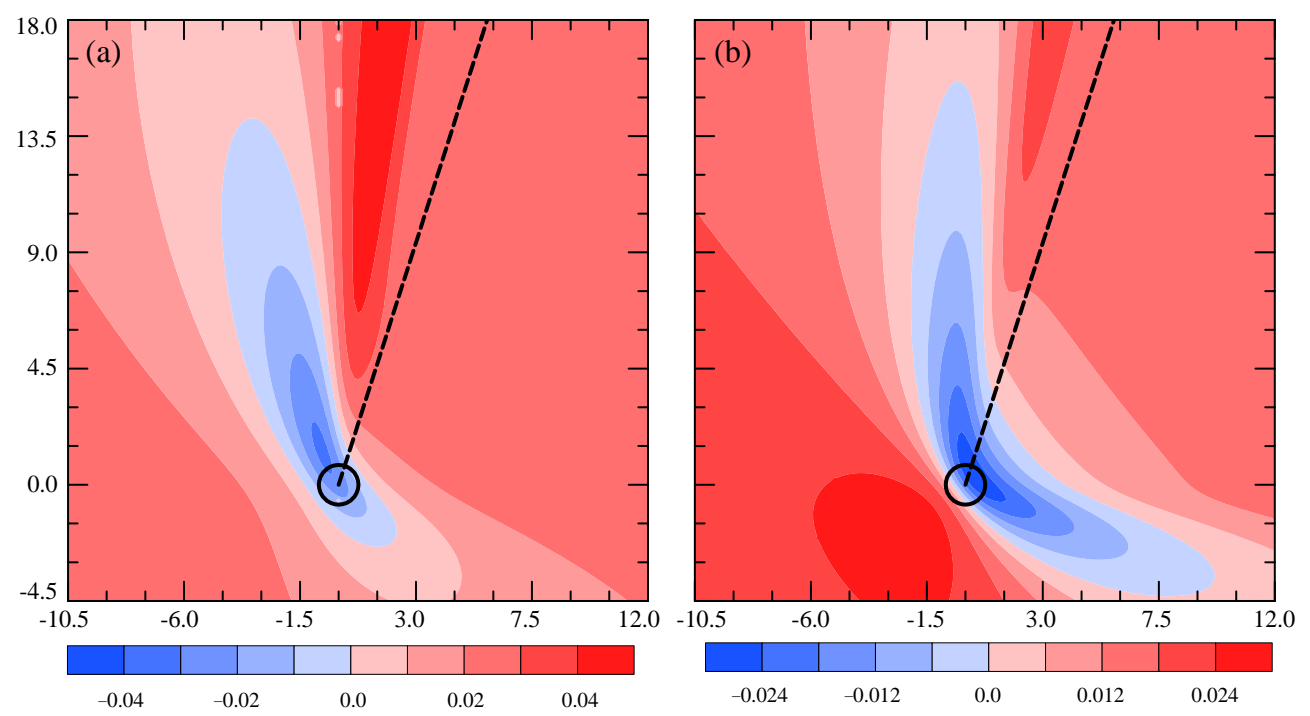

Fig. 4. Perturbation fields of horizontal velocity (a) $u$ (unit: $\mathrm{m} \mathrm{s}^{-1}$ ) and (b) $v$ (unit: $\mathrm{m}$ $\mathrm{s}^{-1}$ ) at the height of $z=5 \mathrm{~km}$. The black circle represents the circular bell-shaped topography. The black lines denote the mean flow direction. The $x$ and $y$ coordinates are normalized with respect to the mountain half width. 

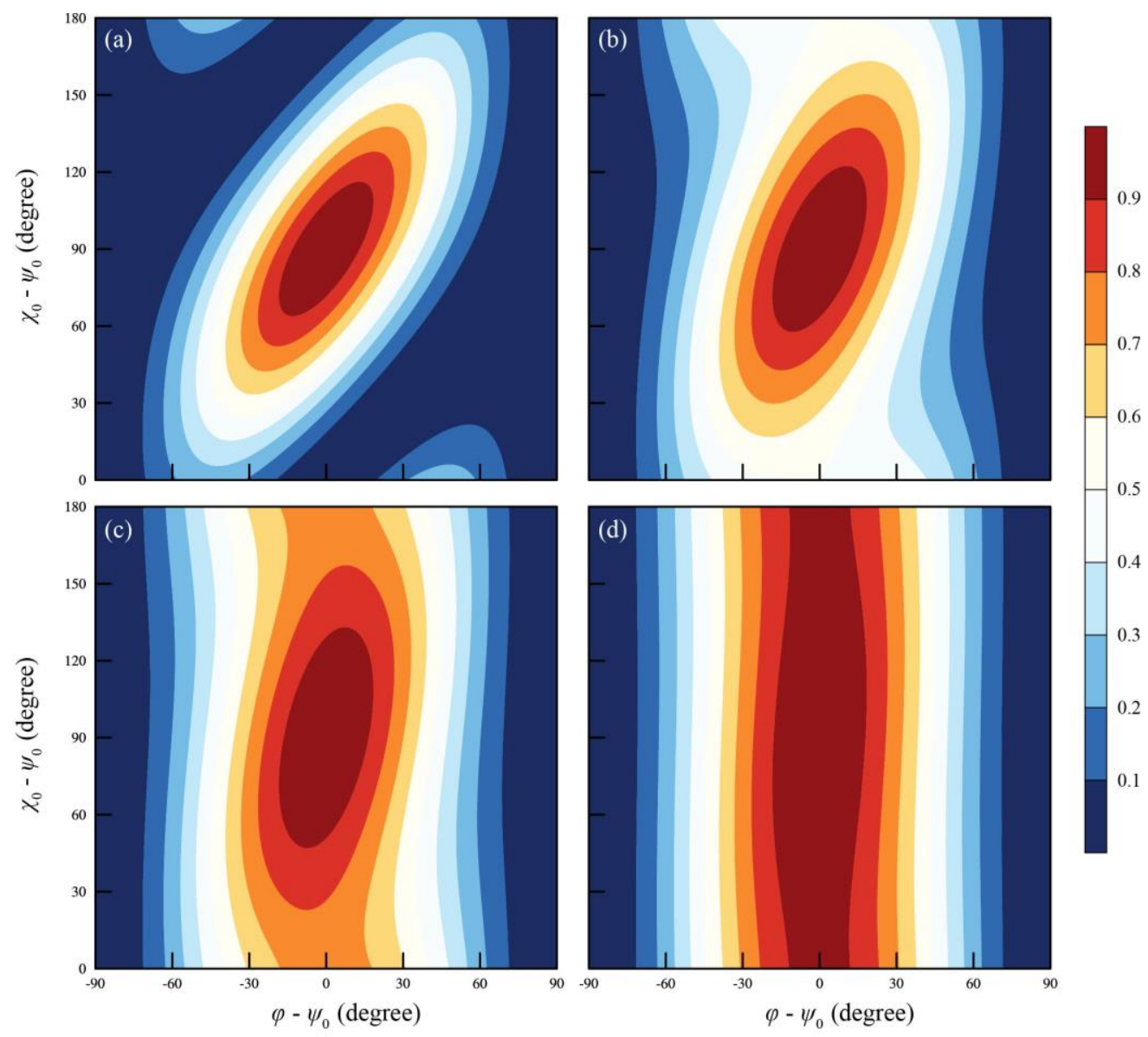

Fig. 5. Distribution of scaled power spectrum of wave momentum flux at (a) $\mathrm{Ri}=$ 0.25, (b) $\mathrm{Ri}=0.5$, (c) $\mathrm{Ri}=1$, and (d) $\mathrm{Ri}=4$. 